\title{
Estructura espacial de remanentes de bosque nativo en el Chaco Seco y el Espinal
}

\author{
Diego Muñoz Garachana ${ }^{1}$; Roxana Aragón² \& Germán Baldi ${ }^{1, \bigotimes}$ \\ ${ }^{1}$ Instituto de Matemática Aplicada San Luis, Universidad Nacional de San Luis - CONICET. San Luis, Argentina. ${ }^{2}$ Instituto \\ de Ecología Regional, Universidad Nacional de Tucumán - CONICET. Yerba Buena, Argentina.
}

\begin{abstract}
Resumen. El vasto territorio del Chaco Seco y el Espinal se consolida como uno de los centros globales de producción de granos y carne. Tanto por distintas normas legales y limitaciones productivas, como por causas culturales, el bosque nativo se preserva en grandes porciones y numerosos pequeños fragmentos inmersos en la matriz agrícola. Estos remanentes suelen pasar desapercibidos para la comunidad científica y para los tomadores de decisiones, y quedan al margen de la planificación territorial y de acciones de conservación. En este trabajo describimos la estructura espacial de los remanentes y generamos un esquema de clasificación que permite identificar distintos tipos según sus propiedades espaciales. Por medio de una estrategia de clasificación basada en objetos, cartografiamos 22000 remanentes en ocho áreas focales (AF) dispersas en el territorio, para lo cual usamos imágenes satelitales CBERS-2B. Mediante índices espaciales, estos remanentes de tamaños, formas y niveles de aislamiento variables fueron categorizados en cinco clases, desde "isletas" hasta "bloques compactos". La superficie de remanentes resultó mayor en el Chaco Seco que en el Espinal (entre $10.1 \%$ y $19.4 \%$ en la primera región, y entre $3.1 \%$ y $7.3 \%$ en la segunda). Las diferencias en el nivel de fragmentación se amplificaron entre AF, dependiendo tanto de la superficie total de remanentes como de la ocurrencia relativa de distintos tipos. Así, en el AF de San Luis, la fragmentación resultó 70 veces superior a la del AF de Anta (Salta). En este trabajo brindamos herramientas para identificar y monitorear estos remanentes; estas herramientas podrán ser consideradas tanto con fines productivos como de conservación. Finalmente, las acciones de manejo y conservación deben reconocer la existencia de estos fragmentos de bosques en paisajes muy transformados por la actividad agropecuaria.
\end{abstract}

[Palabras clave: deforestación, fragmentación, propiedades espaciales, producción agropecuaria, planificación territorial, conservación]

\begin{abstract}
Spatial structure of remnants of native forest in the Dry Chaco and the Espinal. The vast territory of the Dry Chaco and the Espinal became one of the global centers for the production of grains and meat. Due to different legal norms, productive limitations or cultural causes, the native forest is preserved on extensive patches as well as on numerous small fragments within the agricultural matrix. These remnants are seldom considered by the scientific community and decision-makers, and are left out of territorial planning and conservation actions. In this paper, we describe the spatial structure of the remnants and generate a classification scheme that allows identifying different types according to their spatial properties. Following an object-based classification strategy, we mapped 22000 remnants in eight foci areas (FA) scattered throughout the territory using CBERS-2B satellite images. By means of spatial metrics, these remnants of variable sizes, shapes and isolation levels were categorized into five classes, from "islets" to "compact blocks". The surface of remnants was higher in the Dry Chaco than in the Espinal (between $10.1 \%$ and $19.4 \%$ in the first region, and between $3.1 \%$ and $7.3 \%$ in the second). Differences in the level of fragmentation were amplified between FA, depending both on the total surface of remnants and on the relative occurrence of different types. Thus, in the FA of San Luis the fragmentation was 70 times higher than in the FA of Anta (Salta). In this work, we provide tools for the identification and monitoring of these remnants, which may be considered for both productive and conservation purposes. Finally, management and conservation actions must recognize the existence of these pieces of forest in landscapes highly transformed by agricultural activity.
\end{abstract}

[Keywords: deforestation, fragmentation, spatial properties, grain and meat production, land planning, conservation]

Editor asociado: Guillermo Martínez Pastur \baldi@unsl.edu.ar
Recibido: 29 de mayo de 2018

Aceptado: 15 de agosto de 2018 


\section{INTRODUCCIÓN}

Las tierras semiáridas subtropicales del mundo enfrentan una de las transformaciones más grandes de su historia debido a la expansión rápida de cultivos y pasturas sobre la vegetación original de bosques y sabanas (Miles et al. 2006; Baldi and Jobbágy 2012). Entre los factores determinantes de estos cambios se encuentran el aumento de la demanda de alimentos y de biocombustibles de países extrarregionales, la creciente conectividad global entre áreas de producción y de consumo, y la estabilización de sus condiciones económicas (Redo et al. 2011; Gasparri et al. 2016). Las ecorregiones del Chaco Seco y el Espinal, en Sudamérica, no son ajenas a este proceso (Cabrera 1971). Allí, los bosques se vieron afectados notablemente por el avance de la agricultura (Adámoli et al. 2011; Gasparri and Baldi 2013), con tasas anuales de transformación de hasta $4 \%$ anual (Vallejos et al. 2015). Así, la región parece estar destinada a convertirse en uno de los grandes proveedores mundiales de granos y carne, y, al mismo tiempo, en un ejemplo de pérdida de diversidad biológica y desvalorización de los bosques nativos como proveedores de bienes y servicios ecosistémicos.

Tanto por distintas normas legales (e.g., Seghezzo et al. 2011; Collazo et al. 2013), limitaciones productivas (e.g., Fernández et al. 2014) o -aunque menos exploradascausas sociales o culturales (e.g., Mastrangelo et al. 2014), ambas ecorregiones hoy son espacialmente heterogéneas en términos de cobertura, con grandes masas forestales y pequeños remanentes de bosque nativo inmersos en una matriz agrícola compuesta por cultivos anuales y por pasturas de especies megatérmicas (Houspanossian et al. 2016). En este escenario, las grandes porciones de bosque han sido históricamente el foco de la investigación sobre la ecología del Chaco Seco y el Espinal (e.g., Kunst et al. 2006) y el foco de los esfuerzos de conservación (e.g., Senado y Cámara de Diputados de la Nación Argentina 2007). Si bien distintos autores señalan un notable proceso de fragmentación y pérdida de conectividad de los bosques nativos en las últimas décadas (Gasparri and Grau 2009; Carranza et al. 2015; Piquer-Rodríguez et al. 2015), la distribución regional de los pequeños remanentes, así como sus atributos ecológicos y productivos, hoy resultan poco conocidos y son objeto de valoraciones contrapuestas por parte de distintos actores sociales.
Uno de los factores que habría contribuido a su limitado conocimiento estaría relacionado con aspectos tecnológicos y metodológicos del campo científico. La mayoría de los productos cartográficos generados mediante sensores remotos resultan muy adecuados para entender patrones de distribución de bosques a nivel regional (e.g., Hansen et al. 2013). Sin embargo, estos no permiten discriminar objetos de tamaño pequeño en paisajes muy fragmentados (e.g., cortinas forestales), ni describir su disposición espacial o estabilidad temporal. Al presente, y a nivel local, únicamente Ginzburg et al. (2012) realizaron una descripción del proceso de fragmentación - con especial atención en las cortinas forestales-y encontraron un cumplimiento muy bajo de las normas legales, cuestionando su efectividad como medida de conservación del bosque nativo y de provisión de bienes y servicios ecosistémicos.

En relación con las características ecológicas, Núñez-Regueiro et al. (2015) hallaron que las cortinas forestales excluyen especies de mamíferos de gran porte o especialistas, en comparación con el bosque continuo. En tanto, Torrella et al. (2013) describieron que la tasa de reclutamiento de múltiples especies arbóreas se ve muy disminuida, aunque los remanentes mantienen individuos adultos. Ambos trabajos apuntan a una capacidad limitada de los remanentes para mantener las características del bosque y su funcionamiento, y a que la sustentabilidad de estos sistemas se encuentra comprometida. Desde la perspectiva de la provisión de bienes y servicios ecosistémicos, Chacoff y Aizen (2006) encontraron que los remanentes de bosques pedemontanos actúan como fuentes de visitantes florales para plantaciones frutales y que la frecuencia de visitas y la diversidad de visitantes disminuyen con el aumento de la distancia a los remanentes. Por su parte, en un metaanálisis de 10 especies cultivadas, Garibaldi et al. (2011) hallaron que el grado de aislamiento afecta paralelamente la frecuencia de visitas, su estabilidad temporal y la producción de frutos.

En consecuencia, un paso previo para reconocer y ponderar los factores que determinan la calidad estructural y funcional de la vegetación boscosa remanente en paisajes agrícolas y su valor de conservación es reconocer su abundancia y diversidad estructural. Los objetivos de este artículo son 1) describir la estructura espacial de los 
fragmentos remanentes de bosque nativo (de aquí en más, remanentes) en paisajes agrícolas del Chaco Seco y el Espinal, y 2) generar un esquema de clasificación que permita diferenciar tipos de remanentes según sus propiedades espaciales. Para estos fines recurrimos a clasificaciones supervisadas basadas en objetos y al análisis de índices de distintas dimensiones de la estructura espacial de los remanentes como, por ejemplo, su área, elongación o conectividad.

\section{MATERIALES y MÉTODOS}

\section{Región de estudio}

Abarca las ecorregiones del Chaco Seco y el Espinal (Figura 1). El Chaco Seco ocupa una gran extensión de territorio, 1080000 km². Incluye tres países: el centro y norte de la Argentina (60\%), el suroeste de Bolivia (11\%) y el oeste de Paraguay (28\%). Inmediatamente hacia el sur se encuentra el Espinal, un arco de vegetación leñosa de $245000 \mathrm{~km}^{2}$ que se extiende entre las provincias de Chaco y Buenos Aires. En ambas ecorregiones, la ganadería extensiva es la actividad predominante donde el territorio permanece cubierto por bosque (Adámoli et al. 2011) o donde fue reemplazado por pasturas exóticas (e.g., Cenchrus ciliaris, Panicum spp.). El área ocupada por cultivos industriales (i.e., trigo, maíz y particularmente soja) y por pasturas exóticas ocupa actualmente $29 \%$ del territorio y desplaza año a año a la actividad ganadera hacia zonas marginales (van Dam 2003; Houspanossian et al. 2016).

\section{Selección de áreas focales}

El primer paso para determinar las áreas focales (AF) donde la actividad agrícola fuese dominante consistió en recopilar información cartográfica acerca de la distribución de distintos tipos de cobertura. Para tal fin recurrimos a múltiples fuentes, dado que no fue posible contar con un producto actualizado para toda la región. En particular, la información sobre la cobertura boscosa para el Chaco Seco provino de Volante et al. (2012), Vallejos et al. (2015) y Guyra Paraguay (2015), mientras que para el Espinal, de la Unidad de Manejo del Sistema de Evaluación Forestal (UMSEF 2008). Luego, resumimos todas estas capas de información en una grilla de celdas cuadradas de $15 \mathrm{~km}$ de lado, lo cual facilitó la apreciación de los patrones en el espacio geográfico. Por último, seleccionamos

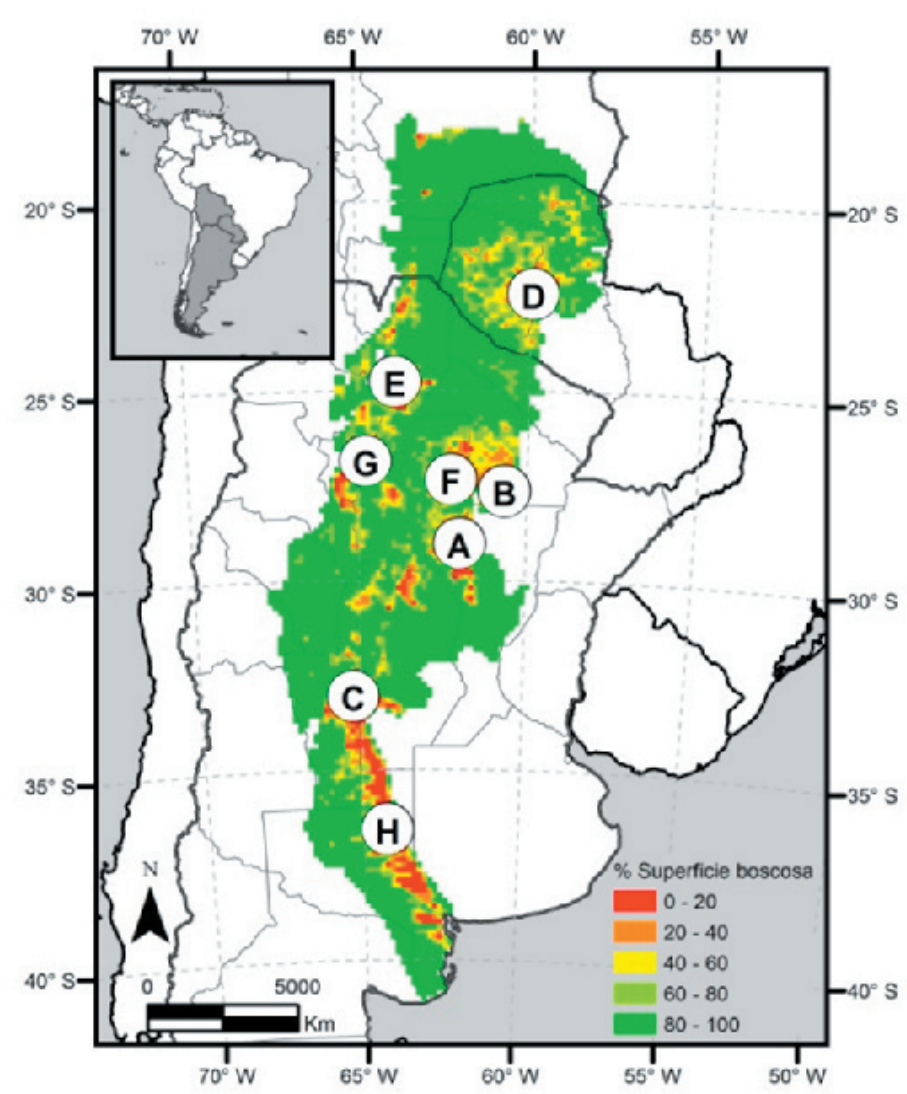

Figura 1. Región de estudio. Se representan las ecorregiones del Chaco Seco y el Espinal, las 8 áreas focales (A: Bandera, B: Charata, C: San Luis, D: Filadelfia, E: Anta, F: Moreno, G: Las Cejas, H: La Pampa) y el nivel de deforestación en celdas cuadradas de $15 \mathrm{~km}$ de lado.

Figure 1. Study region. Represented are the Dry Chaco and Espinal ecoregions, the 8 foci areas (A-Bandera, B-Charata, C-San Luis, D-Filadelfia, E-Anta, F-Moreno, G -Las Cejas, and H - La Pampa) and the level of deforestation in square cells of $15 \mathrm{~km}$ side. 
aquellas celdas que cumplieran los criterios de ser preponderantemente agrícolas $(>75 \%)$, estar categorizadas mayoritariamente $(>80 \%)$ como Categoría III (verde) según la Ley $\mathrm{N}^{\circ}$ 26331 (Senado y Cámara de Diputados de la Nación Argentina 2007) en la Argentina, y formar un conjunto de celdas contiguas con una superficie mayor de $2025 \mathrm{~km}^{2}$ (equivalente a 9 celdas). Las áreas Categoría III (verde) son aquellas de mayor aptitud agrícola, independientemente de su valor ecológico. La única estrategia de conservación en ellas consiste en dejar pequeñas reservas, clausuras, o cortinas forestales con el fin de interconectar las grandes masas de bosque nativo de las áreas Categoría II (amarillas) o Categoría I (rojas) (Ginzburg et al. 2012).

\section{Clasificación de los remanentes}

En las ocho AF delimitadas identificamos los remanentes por medio de clasificaciones supervisadas con imágenes de mayor resolución espacial a la utilizada en los productos cartográficos preexistentes (i.e., $<30 \mathrm{~m}$ ). Las imágenes empleadas provinieron de los sensores pancromáticos HRC (cámara pancromática de alta resolución) y multiespectrales CCD (cámara de alta resolución) a bordo del satélite CBERS-2B (satélite chino-brasileño de recursos terrestres). Las imágenes HRC se caracterizan por una resolución espacial de $2.7 \mathrm{~m}$ y un ancho de barrido de $27 \mathrm{~km}$. El sensor HRC opera en una única banda espectral que cubre el visible y parte del infrarrojo cercano. Las imágenes CCD se caracterizan por capturar información en cinco bandas espectrales, por poseer una resolución espacial de $20 \mathrm{~m}$ y un ancho de barrido de $113 \mathrm{~km}$. Dadas las diferencias entre sensores, son necesarios cinco ciclos de 26 días para que el instrumento HRC capture información para una escena completa provista por el sensor CCD. Por ello, en primer lugar generamos mosaicos de imágenes HRC que coincidieran con cada imagen CCD y luego integramos estos dos productos satelitales mediante el proceso conocido como pansharpening, que implica la fusión de una imagen multiespectral de, comparativamente, menor resolución (i.e., CCD) y una banda pancromática de, comparativamente, mayor resolución (i.e., HRC) (Vijayaraj et al. 2004). Por lo tanto, la imagen que generamos y usamos en la clasificación posee la resolución espacial de la banda pancromática y la radiométrica de la multiespectral. Se emplearon 20 imágenes para HRC y 8 CCD (una por cada AF) en el período 01/09/2008 - 21/01/2010.

El método de clasificación seleccionado se basó en la segmentación de las imágenes y la clasificación de objetos (OBIA, del inglés object based image analysis) de forma supervisada por medio del software ENVI EX 5.0 (Exelis Visual Information Solutions, Boulder, Colorado). Para todas las AF, los niveles de segmentación y fusión empleados fueron $70 \%$ y $95 \%$, respectivamente. El método OBIA es capaz de ofrecer mejores resultados que los obtenidos por un enfoque tradicional basado sobre píxeles (clasificación supervisada y no supervisada), en particular cuando se trata de imágenes de alta o muy alta resolución espacial en territorios de estructura compleja (Hay et al. 2005; Chen et al. 2015). En cada una de las

Tabla 1. Índices empleados para describir la estructura del paisaje y tipificar los remanentes de bosque nativo. El tamaño efectivo de malla (TEM) puede considerarse como un índice sintético de la fragmentación de un paisaje, al incorporar simultáneamente el tamaño de los parches y el nivel de disección del paisaje, y ser insensible a la inclusión u omisión de pequeños parches (Jaeger 2000). $A_{i}$ : área del remanente $i, A_{t}$ : área total del área focal, $P_{i}$ : perímetro del remanente $i$, $n$ : número de remanentes del área focal, E: eje de mayor tamaño del remanente i, e: eje de menor tamaño del remanente i.

Table 1. Metrics used to describe the structure of the landscape and to typify the remnants of native forest. The effective mesh size (TEM) can be considered as a synthetic metric of landscape fragmentation, by simultaneously incorporating the patch size and the level of landscape dissection, and by being insensitive to the inclusion or omission of small patches (Jaeger 2000). Acronyms: $A_{i}$ area of the $i$ remnant, $A_{t}$ total area of the focal area, $P_{i}$ perimeter of the $i$ remnant, $n$ number of remnants of the focal area, $E$ largest size axis of the $i$ remnant, $e$ smallest size axis of the $i$ remnant.

\begin{tabular}{|c|c|c|c|c|}
\hline \# & Índice de paisaje & Unidades & $\begin{array}{l}\text { Acrónimo y fórmula a nivel } \\
\text { área focal }\end{array}$ & $\begin{array}{l}\text { Fórmula a nivel } \\
\text { remanente }\end{array}$ \\
\hline 1 & Porcentaje de superficie & $\%$ & $\mathrm{PS}=100 *\left[\left(\sum_{\mathrm{i}=1->n} \mathrm{~A}_{\mathrm{i}}\right) / \mathrm{A}_{\mathrm{t}}\right]$ & No calculado \\
\hline 2 & Tamaño efectivo de malla & ha & $\mathrm{TEM}=1 / \mathrm{A}_{\mathrm{t}} * \sum_{\mathrm{i}=1>\mathrm{n}} \mathrm{A}_{\mathrm{i}}^{2}$ & No calculado \\
\hline 3 & Densidad & remanente/100 ha & Den $=1 / A_{t} * \sum_{i=1->n} P_{i}$ & No calculado \\
\hline 4 & Tamaño & ha & $\operatorname{Tam}=\left(\sum_{\mathrm{i}=1>\mathrm{n}} \mathrm{A}_{\mathrm{i}}\right) / \mathrm{n}$ & $\operatorname{Tam}=\mathrm{A}_{\mathrm{i}}$ \\
\hline 5 & Distancia euclidiana al vecino más cercano & $\mathrm{m}$ & $\mathrm{DVC}=\left(\sum_{\mathrm{i}=1-\mathrm{n}} \mathrm{H}_{\mathrm{i}, \mathrm{i}+1}\right) / \mathrm{n}$ & $\mathrm{DVC}=\mathrm{H}_{\mathrm{i}, \mathrm{i}+1}$ \\
\hline 6 & Relación perímetro-área & $1 / \mathrm{m}$ & No calculado & $\mathrm{RPA}=\mathrm{P}_{\mathrm{i}} / \mathrm{A}_{\mathrm{i}}$ \\
\hline 7 & Longitud & $\mathrm{m}$ & No calculado & Lon $=E_{i}$ \\
\hline 8 & Elongación & - & No calculado & Elo $=\mathrm{E}_{\mathrm{i}} / \mathrm{e}_{\mathrm{i}}$ \\
\hline
\end{tabular}


ocho AF seleccionamos de manera visual 100 muestras (celdas cuadradas de $20 \mathrm{~m}$ de lado) para cada clase de información, bosque nativo y misceláneos; de éstas, una mitad la usamos para entrenar el algoritmo de clasificación y la otra mitad para evaluar la clasificación. Para la evaluación calculamos el número total de píxeles correctamente clasificados de cada categoría dividido el número total de píxeles de esa categoría derivado de los datos de referencia. Esta precisión indica la probabilidad de que un píxel de referencia de una determinada cobertura sea correctamente clasificado, medida denominada precisión del productor (Congalton 1991).

\section{Caracterización estructural}

A partir de la información de tipo vectorial de la clase bosque nativo estimamos ocho índices que describen de forma cuantitativa la estructura espacial de 1) el AF en general (e.g., índice tamaño efectivo de malla) y 2) cada uno de los remanentes en particular (e.g., índice elongación) (Tabla 1) (Riitters et al. 1995). Para tipificar los remanentes a partir de los índices a nivel remanente generamos un esquema de clasificación conceptual basado en tipos reconocidos de remanentes (e.g., Ginzburg et al. 2012). Obtuvimos cinco tipos: Bloque compacto (i.e., parche de bosque sin signos de fragmentación ni deterioro), Bloque perforado (i.e., parche de bosque con signos de degradación e inicio de fragmentación), Cortina forestal (i.e., parche de bosque alargado), Resto de cortina (i.e., parche de bosque pequeño y cercano a cortinas forestales), e Isleta (i.e., parche de bosque pequeño y aislado, o árboles individuales). A partir de esta clasificación conceptual tipificamos cada uno de los remanentes mediante un árbol de decisión ad hoc (Figura 2). Aplicamos los índices sobre todas las clases en su conjunto e individualmente. Los índices provienen del Patch Analyst v.5 para ArcGIS 10 (Rempel et al. 2012) y del Fragstat v.4.2.1 (McGarigal et al. 2015).

\section{Resultados}

Por medio de una estrategia de clasificación basada en objetos sobre imágenes CBERS-2B del Chaco Seco y el Espinal, discriminamos 21758 remanentes de tamaños, formas y niveles de aislamiento variables en los $12854 \mathrm{~km}^{2}$ que analizamos. Este bosque remanente en paisajes agrícolas, que en términos globales ocupa $11.7 \%$ de la superficie (Tabla 2), no suele ser

Tabla 2. Estructura del paisaje en las ocho áreas focales de la región de estudio. Los valores representan el promedio y, exclusivamente para los índices calculados a nivel remanente, su desvío estándar (entre paréntesis). A nivel paisaje se incluyen todos los remanentes de bosques nativos. TEM: tamaño efectivo de malla.

Table 2. Landscape structure in the eight 8 areas of the study region. The values represent the average and, exclusively for the metrics calculated at the remnant level, their standard deviation -between parentheses. At the landscape level, all remnants of native forests are included. Acronym: TEM effective mesh size.

\begin{tabular}{|c|c|c|c|c|c|c|c|c|c|c|c|c|c|c|c|c|c|c|c|c|c|c|c|}
\hline \multirow{2}{*}{ 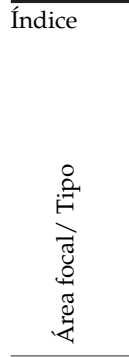 } & \multicolumn{6}{|c|}{$\begin{array}{l}\text { Porcentaje de superficie } \\
\qquad(\%)\end{array}$} & \multirow[b]{2}{*}{ 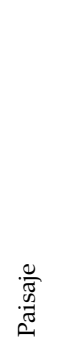 } & \multirow[b]{2}{*}{ 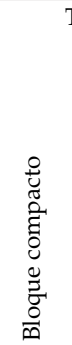 } & \multicolumn{3}{|c|}{ Tamaño (ha) } & \multirow[b]{2}{*}{ 吾 } & \multicolumn{6}{|c|}{$\begin{array}{l}\text { Densidad (remanente/100 } \\
\text { ha) }\end{array}$} & \multicolumn{5}{|c|}{$\begin{array}{l}\text { Distancia euclidiana al TEM } \\
\text { vecino más cercano }\end{array}$} \\
\hline & & 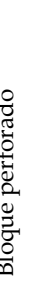 & 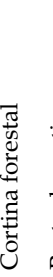 & 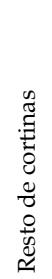 & $\frac{\frac{\pi}{0}}{9}$ & & & 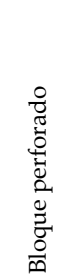 & 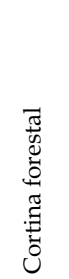 & 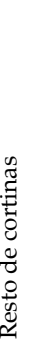 & & 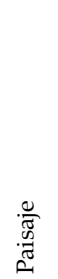 & 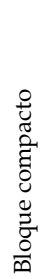 & 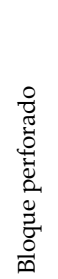 & 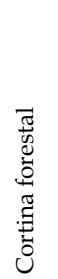 & 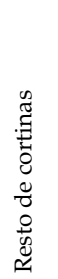 & $\frac{\frac{\pi}{0}}{\sqrt{0}}$ & 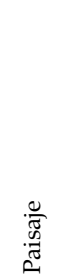 & 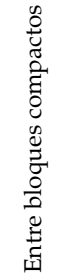 & 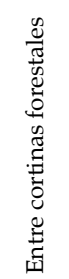 & 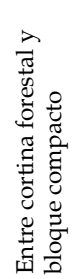 & 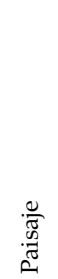 \\
\hline . & 4 & 9.0 & 1.4 & 8 & 24 & ( & $\begin{array}{c}10.3 \\
(160.6)\end{array}$ & $\begin{array}{c}338 \\
(1325)\end{array}$ & $\begin{array}{c}8.1 \\
(18.7)\end{array}$ & $\begin{array}{c}7.2 \\
(16.4)\end{array}$ & & $\begin{array}{c}0.45 \\
(0.22)\end{array}$ & 1.89 & 0.03 & 0.18 & 1.21 & 0.37 & 10 & $\begin{array}{c}69 \\
(149)\end{array}$ & $\begin{array}{c}1071 \\
(1817)\end{array}$ & $\begin{array}{c}86 \\
(134)\end{array}$ & $\begin{array}{c}107 \\
(332)\end{array}$ & 488 \\
\hline ndera & 10.1 & 7.0 & 1.1 & 1.81 & 0.06 & 0.14 & $\begin{array}{c}6.8 \\
(37.5)\end{array}$ & $\begin{array}{l}06 \\
45)\end{array}$ & .0 & $\begin{array}{l}6 \\
3)\end{array}$ & & & 1.49 & 0.07 & 0.38 & 0.50 & 0.09 & 0.45 & $\begin{array}{l}141 \\
(222)\end{array}$ & $\begin{array}{l}801 \\
(875)\end{array}$ & $\begin{array}{c}241 \\
(375)\end{array}$ & $\begin{array}{c}304 \\
(486)\end{array}$ & 22 \\
\hline arata & 16.6 & 12.4 & 3.5 & 0.48 & 0.02 & 0.18 & $\begin{array}{c}6.9 \\
(33.5)\end{array}$ & & $4)$ & & & & 2.40 & 16 & 1.09 & 0.28 & 0.12 & 0.76 & $\begin{array}{l}104 \\
(170)\end{array}$ & $\begin{array}{c}335 \\
(448)\end{array}$ & $\begin{array}{c}324 \\
(535)\end{array}$ & & 8 \\
\hline delfia & 7 & 8.7 & 1.9 & 4.03 & 0.14 & 0.87 & $\begin{array}{c}3.3 \\
(78.2)\end{array}$ & & & $\begin{array}{c}2.8 \\
(14.9)\end{array}$ & & & 4.81 & 006 & 0.18 & 1.43 & 0.372 & 2.78 & $\begin{array}{c}129 \\
(316)\end{array}$ & $\begin{array}{c}1108 \\
(1389)\end{array}$ & $\begin{array}{c}184 \\
(397)\end{array}$ & $\begin{array}{l}218 \\
\text { (548) }\end{array}$ & 134 \\
\hline $\mathrm{a}$ & 7.3 & 7.0 & 0.2 & - & - & 0.00 & & & & & - & & & & 0.07 & - & ( & .02 & $\begin{array}{c}312 \\
(834)\end{array}$ & & - & - & 48 \\
\hline Cejas & 14.0 & 11.7 & 1.2 & 0.98 & 0.04 & 0.06 & $\begin{array}{c}9.6 \\
(63.9)\end{array}$ & & $\begin{array}{c}2.8 \\
(8.0)\end{array}$ & $\begin{array}{c}2.4 \\
(4.5)\end{array}$ & & & 1.46 & 0.09 & 0.44 & 0.42 & 0.11 & 0.40 & $\begin{array}{c}90 \\
(188)\end{array}$ & $\begin{array}{c}442 \\
(645)\end{array}$ & $\begin{array}{c}133 \\
(318)\end{array}$ & $\begin{array}{c}161 \\
(385)\end{array}$ & 61 \\
\hline Ioreno & 1.1 & 8.5 & 4 & & 0.22 & 0.06 & $\begin{array}{c}16.7 \\
(110.1)\end{array}$ & 240 & & & & & & 0.04 & 0.12 & 43 & 0.16 & 0.10 & $\begin{array}{c}131 \\
(146)\end{array}$ & $\begin{array}{c}1366 \\
(1436)\end{array}$ & $\begin{array}{c}142 \\
(263)\end{array}$ & $\begin{array}{c}232 \\
(454)\end{array}$ & 35 \\
\hline uis & 3.1 & 1.7 & 1.1 & 0.14 & 0.02 & 0.12 & & & $\begin{array}{c}1.5 \\
(8.6)\end{array}$ & $\begin{array}{c}2.4 \\
(6.0)\end{array}$ & & & 1.8 & 0.02 & 0.75 & 0.06 & 0.04 & 0.95 & $\begin{array}{c}82 \\
(370)\end{array}$ & $\begin{array}{l}76 \\
447)\end{array}$ & $\begin{array}{c}420 \\
(784)\end{array}$ & 5 & 7 \\
\hline omedio & 11.7 & 7.5 & 1.5 & 2.90 & 0.11 & 0.18 & 3.0 & 160 & 5.6 & 4.2 & 67 & 0.32 & 1.86 & 0.07 & 0.40 & 0.62 & 0.18 & 0.70 & 132 & 777 & 219 & 266 & 105 \\
\hline
\end{tabular}




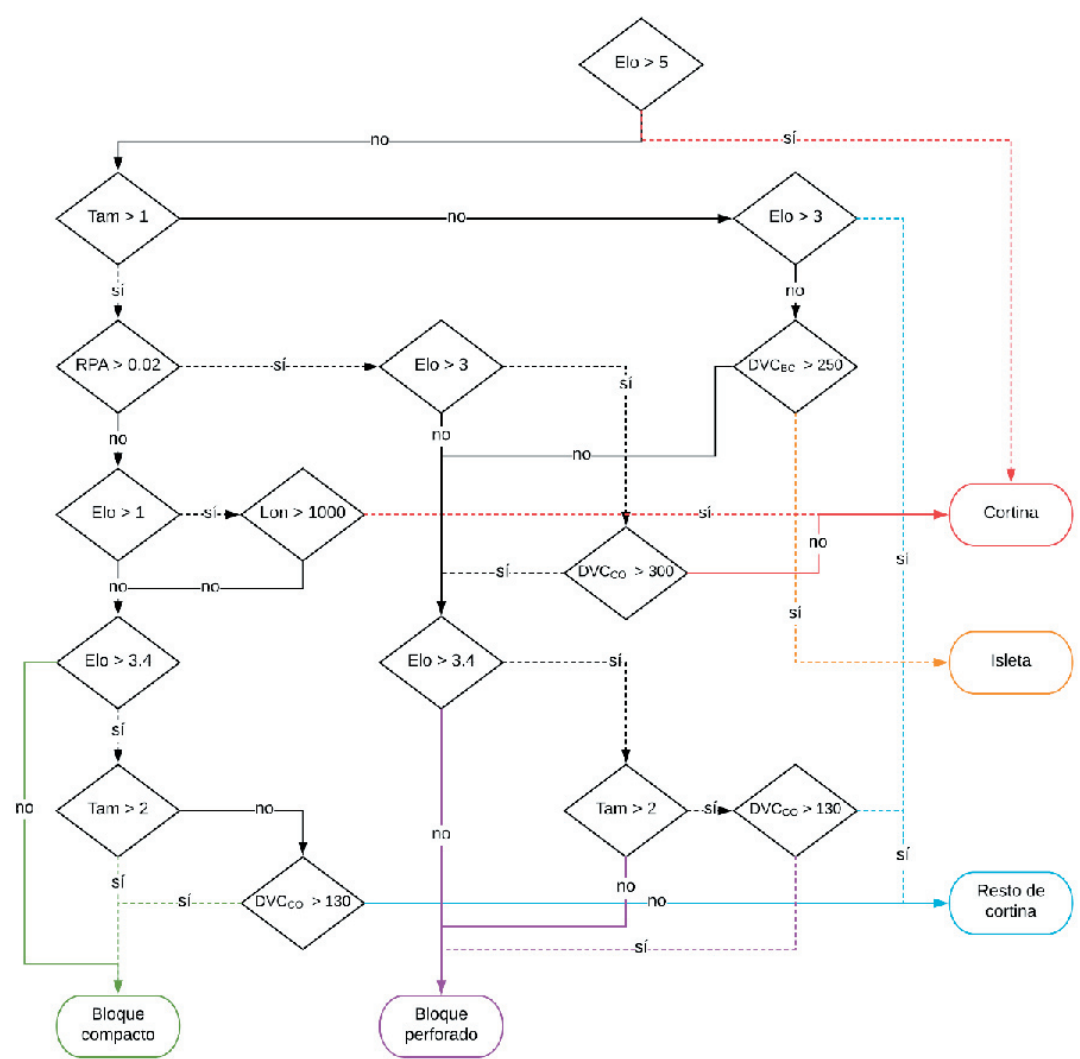

Figura 2. Árbol de decisión para tipificar remanentes de bosque nativo. Los rombos indican decisiones, mientras que las elipses indican terminadores (i.e., los tipos). Los conectores se diferencian según si son negativos (línea llena) o positivos (línea punteada) o si conducen a un determinado terminador, por color. BC: bloque compacto, CO: cortina forestal, DVC: distancia euclidiana al vecino más cercano, Elo: elongación, Lon: longitud, RPA: relación perímetroárea, Tam: tamaño.

Figure 2. Decision tree used to typify remnants of native forest. The diamonds indicate decisions, while the ellipses indicate terminators (i.e., types). The connectors are differentiated according to whether they are negative (full line) or positive (dotted line) and depending on whether they lead to a specific terminator, by color. Acronyms: BC compact block, CO forest strip, DVC Euclidean distance to nearest neighbor, Elo elongation, Lon length, RPA perimeter-area ratio, Tam size.
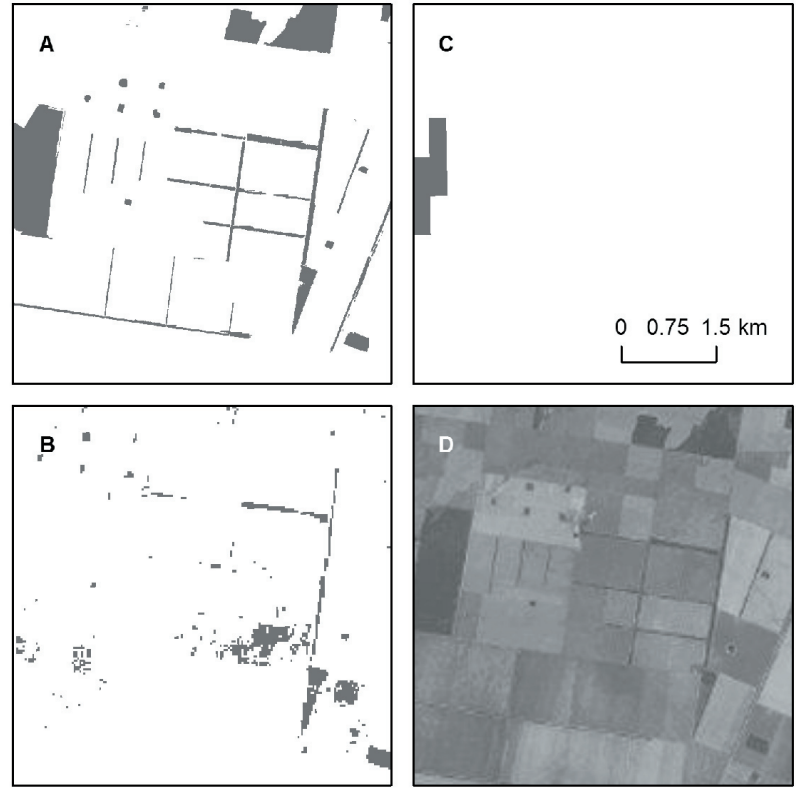

Figura 3. Detalle de remanentes de bosque nativo (en gris) en el área focal de Las Cejas a partir de (A) la clasificación 2008-2010 basada en objetos empleando imágenes C-BERS 2B de este trabajo, (B) una clasificación basada en características espectrales empleando imágenes Landsat ETM+ circa 2000 (Hansen et al. 2013), (C) una clasificación basada en características espectrales empleando imágenes MERIS circa 2004 (Bicheron et al. 2008) y (D) una imagen DigitalGlobe de muy alta resolución de Google Earth.

Figure 3. Detail of native forest remnants (in gray) in the focal area of Las Cejas from (a) the 20082010 object-based classification using C-BERS 2B images used in this study, (b) a classification based on spectral characteristics using Landsat ETM+ circa 2000 images (Hansen et al., 2013), (c) a classification based on spectral characteristics using MERIS images circa 2004 (Bicheron et al., 2008) and (d) a very high resolution DigitalGlobe image of Google Earth. 
detectado en las clasificaciones convencionales generadas a partir de imágenes de alta a media resolución espacial, tales como Landsat o Meris (Figura 3). Aproximadamente, existen 2 remanentes por cada 100 ha; el tamaño promedio de los remanentes es 13 ha y están conectados entre sí por una distancia media de $132 \mathrm{~m}$.

Al explorar la variabilidad estructural de las AF encontramos diferencias notables entre ecorregiones en relación a la superficie bajo bosque nativo (Tabla 2). El Espinal, representado por las AF de La Pampa y San Luis, mantiene una superficie de bosque pequeña (PS $<7.3 \%$ ), comparado con el Chaco Seco (PS $>10.1 \%$ ). Dentro del Chaco Seco no existen diferencias con respecto al país, ya que el AF de Paraguay se posiciona en el medio del rango de las AF de Argentina (i.e., $10.1 \%>$ PS $_{\text {Argentina }}<19.4 \%$ ). El tamaño efectivo de malla, indicador sintético del grado de cohesión de una clase de cobertura (en este caso, de bosque nativo) muestra cierta similitud con el porcentaje de la superficie con remanentes, al repetirse los extremos (i.e., Anta, con los mayores valores de ambos índices en relación a la situación inversa, en San Luis). No obstante, las diferencias se amplifican en el tamaño efectivo de malla, con valores para Anta 3.6 veces más altos que en Filadelfia (segunda AF en el ranking, ambas en el Chaco Seco) y 70 veces más altos que en San Luis.

La variabilidad en el tamaño efectivo de malla dependería tanto de la superficie bajo bosque como de la ocurrencia relativa de distintos tipos de remanentes (Tabla 2). Así distinguimos que, en Anta, los bloques compactos y las cortinas forestales ocupan una fracción alta del AF (PS 8.9\%), con una configuración de pocos bloques compactos de gran tamaño (Den $=0.03$ remanentes $/$ ha y Tam=338 ha), muchas cortinas forestales (Den $=1.21$ remanentes $/$ ha y Tam $=7.2$ ha) y una distancia entre remanentes de cualquier tipo baja $(\mathrm{DVC}=69 \mathrm{~m})$. Esto resulta en un paisaje donde la cobertura boscosa, aunque reducida, se encuentra relativamente bien interconectada (Figura 4). En el extremo opuesto se pueden describir AF con bloques compactos o perforados de $\sim 110$ ha $y$ sin cortinas forestales o restos de ellas (en San Luis y La Pampa), o con bloques compactos de menor tamaño y con cortinas forestales aisladas entre sí (en Bandera o Charata, 241 m>DVC $<324$ m) (Figura 4). La Pampa, el AF más austral del Espinal, en particular presenta la distancia más elevada entre todos los tipos de remanente $(\mathrm{DVC}=312 \mathrm{~m})$. Las $\mathrm{AF}$ de Las Cejas y Moreno (ambas en la Argentina) y de Filadelfia (en Paraguay), presentan paisajes comparativamente en una posición intermedia entre los escenarios antes descriptos, donde Moreno es el AF con las cortinas forestales de mayor extensión (Tam=9 ha). Cabe destacar que los resultados antes expuestos surgen de productos cartográficos con niveles de

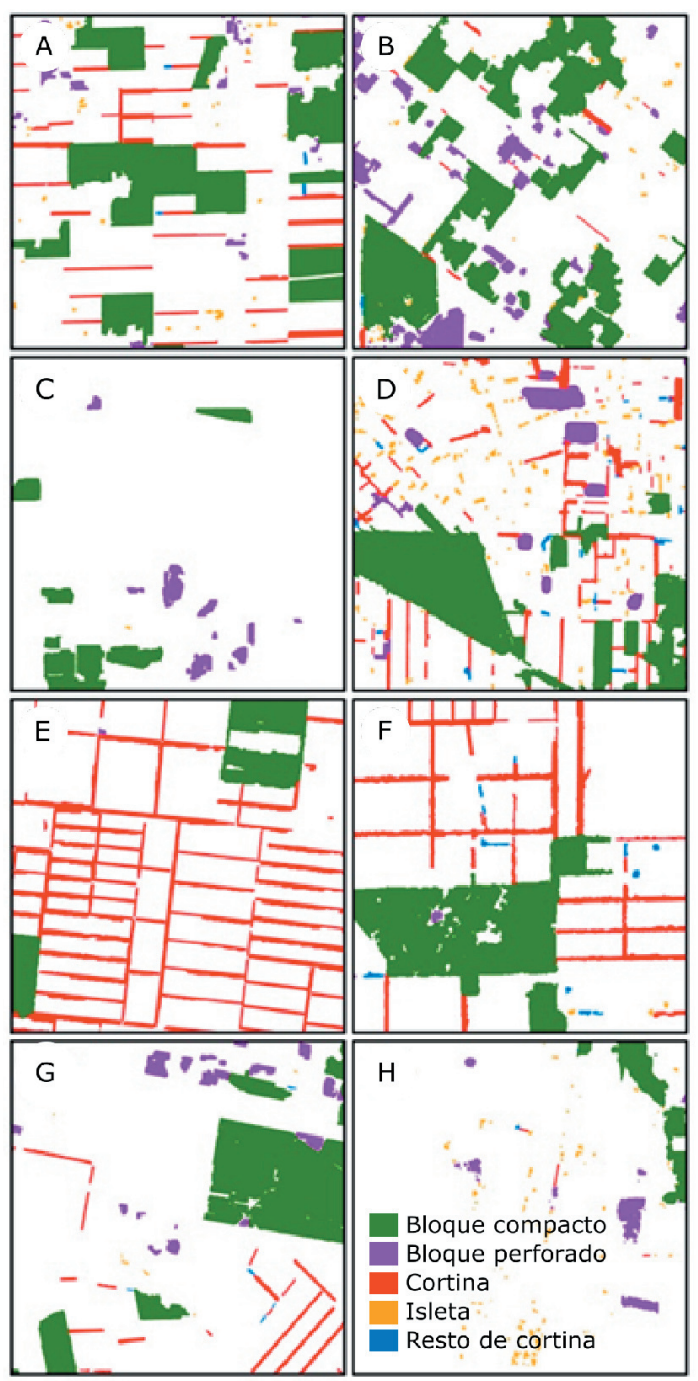

Figura 4. Ejemplos de paisajes en las 8 áreas focales, discriminando los remanentes de bosque nativo según su tipo. (A) Bandera, (B) Charata, (C) La Pampa, (D) Filadelfia, (E) Anta, (F) Las Cejas, (G) Moreno y (H) San Luis.

Figure 4. Examples of landscapes in the 8 foci areas, discriminating the remnants of native forest according to their type. (A) Bandera, (B) Charata, (C) La Pampa, (D) Filadelfia, (E) Anta, (F) Las Cejas, (G) Moreno and (H) San Luis. 
Tabla 3. Precisión del método de clasificación de imágenes por OBIA.

Table 3. Accuracy of the image classification method from OBIA.

\begin{tabular}{lcccc}
\hline $\begin{array}{l}\text { Área } \\
\text { focal }\end{array}$ & $\begin{array}{c}\text { Error bosque } \\
\text { nativo }(\%)\end{array}$ & $\begin{array}{c}\text { Error } \\
\text { misceláneos }(\%)\end{array}$ & $\begin{array}{c}\text { Error total } \\
(\%)\end{array}$ & $\begin{array}{c}\text { Precisión } \\
(\%)\end{array}$ \\
\hline Anta & 2 & 0 & 2 & 98 \\
Bandera & 5 & 0 & 5 & 95 \\
Charata & 3 & 0 & 3 & 97 \\
Filadelfia & 8 & 1 & 9 & 91 \\
La Pampa & 4 & 2 & 6 & 94 \\
Las Cejas & 2 & 3 & 5 & 95 \\
Moreno & 6 & 0 & 6 & 94 \\
San Luis & 2 & 0 & 2 & 98 \\
\hline
\end{tabular}

precisión general altos, que oscilan entre $94 \%$ y $98 \%$ (Tabla 3).

\section{DisCUSIÓN}

Las acciones de manejo y conservación en el Chaco Seco y el Espinal deben reconocer la existencia de una extensa superficie boscosa remanente en paisajes muy transformados por la actividad agropecuaria. En este trabajo identificamos estos fragmentos remanentes de bosque nativo por medio de clasificaciones basadas en objetos sobre imágenes de muy alta resolución espacial y describimos su estructura espacial mediante una serie de índices (Tabla 2) que nos permiten por último tipificar la variabilidad existente (Figura 2). El producto cartográfico generado supone una mejora significativa en la detección de estos remanentes con respecto a otros de aceptación y empleo generalizados (e.g., Bicheron et al. 2008; Hansen et al. 2013) (Figura 3), por lo que la metodología posee una aplicación potencial en territorios de estructura espacial compleja dentro y fuera de la región de estudio.

Recientemente, Piquer-Rodríguez et al. (2015) describieron para las ecorregiones del Chaco Seco y Húmedo un retroceso fuerte de la cobertura boscosa y un incremento del aislamiento de los bosques nativos (período 1977-2010). Este aumento en el número de remanentes implica que el territorio se transformó de manera radical; su matriz pasó de estar constituida por bosques nativos a estarlo por cultivos y pasturas. Considerando este tipo de transformaciones, dos décadas atrás, Forman (1995) y Jaeger (2000) tipificaron distintas fases de pérdida de hábitat y fragmentación creciente denominadas de incisión, perforación, disección, disipación, encogimiento y agotamiento. En nuestro trabajo ratificamos que, aunque diversos, los patrones estructurales de las ocho AF representan condiciones de alto nivel de pérdida de hábitat y fragmentación (etapas de disipación hasta agotamiento) bajo las cuales los remanentes pierden tamaño o simplemente desaparecen (Figuras 4 y 5a). Esta situación se manifiesta más fuertemente en el Espinal. Sus dos AF analizadas presentaron la menor superficie relativa bajo bosque y una gran desconexión entre sus remanentes (dada en parte por la ausencia de cortinas forestales). Bajo las condiciones descriptas en este trabajo, resulta fundamental reconocer el valor de cada pequeño bloque, cortina e isleta al brindar conexión entre las grandes masas forestales aún existentes, al constituir el espacio que potencialmente proveerá servicios ecosistémicos a la población y donde las especies nativas persistirán. Las condiciones que brindan los remanentes, así como las funciones que cumplen, son múltiples (e.g., disponibilidad de alimento para la fauna) (Tabarelli et al. 2010), por lo que entendemos que futuros estudios deberían establecer umbrales de respuesta o funciones de afectación en relación al nivel de fragmentación de paisaje en el que están inmersos.

Numerosas publicaciones tanto en revistas nacionales o internacionales evaluaron el efecto de distintas propiedades espaciales de los remanentes de vegetación nativa sobre su calidad, dada por la riqueza y la abundancia de especies leñosas, la densidad de individuos, la regeneración de especies clave, la biomasa, la productividad primaria aérea o la estructura vertical (e.g., Chacoff et al. 2004; Torrella et al. 2013) (Figura 5b). Sin embargo, y notablemente, las investigaciones ecológicas y productivas de estos sistemas raramente incluyen en modelos cuantitativos al legado de usos anteriores dentro del propio remanente, al tiempo transcurrido en condiciones de aislamiento, así como al contexto productivo o cultural en el que están inmersos. Según nuestra percepción, la no consideración de los efectos de estos factores obstaculizaría la identificación de tipos de manejo más conservativos para estos ambientes.

En este sentido, la teoría ecológica indica que el tamaño y la forma de los remanentes determinan el grado de influencia ambiental que ejerce la matriz (e.g., modificaciones en temperatura y radiación), hecho que se manifiesta claramente en los bordes del remanente (Saunders et al. 1991; Krauss et al. 2004). El contexto productivo o cultural 

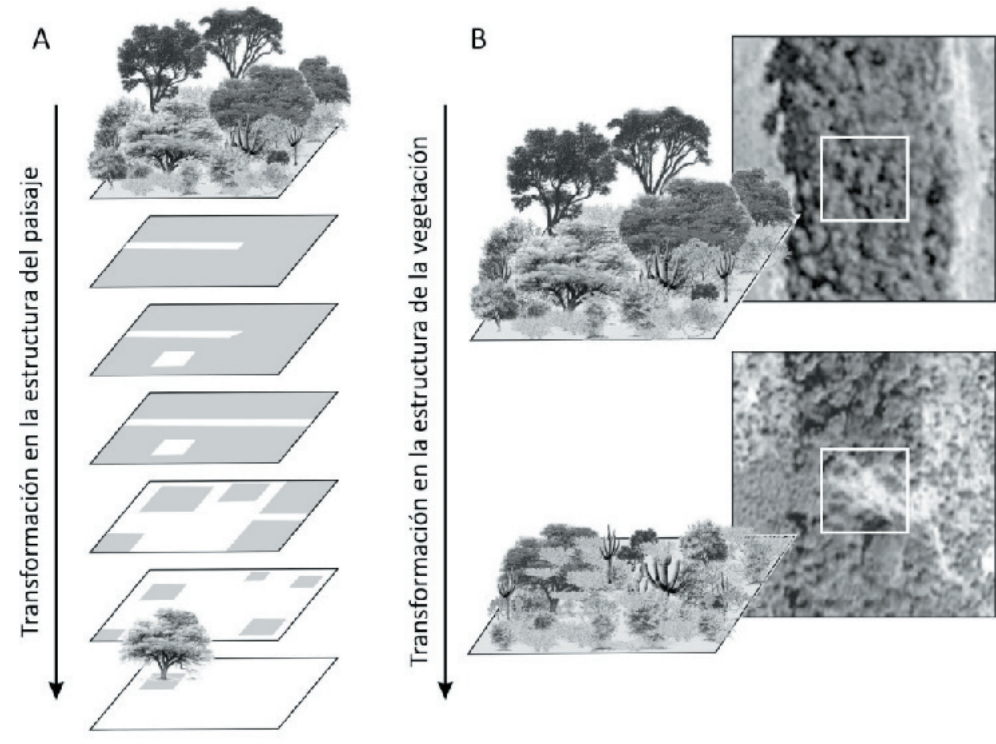

Figura 5. (A) Esquema de distintas etapas del proceso de fragmentación del paisaje de bosques secos (bosques en gris, cultivo o pastura en blanco). (B) Esquema de modificación estructural de un remanente de bosque nativo. Las imágenes constituyen un ejemplo de cortinas forestales en la provincia de Santiago del Estero (imágenes DigitalGlobe de muy alta resolución de Google Earth).

Figure 5. (a) Scheme of different stages of the fragmentation process of a dry forest landscape (forests in gray, crop or pasture in white). (b) Scheme of a structural modification of a remnant of native forest. The images are an example of forest strips in the province of Santiago del Estero (very high resolution DigitalGlobe images from Google Earth).

determinaría de forma directa el tipo de intervención sobre el remanente (e.g., cosecha de leña, pastoreo) y, por lo tanto, su composición de especies, las propiedades del suelo o el régimen natural de disturbios (Macchi and Grau 2012; Rueda et al. 2015). El tipo de uso de la matriz (i.e., cultivos vs. pasturas), la capacidad de acceso a insumos de los actores locales (e.g., fertilización, uso de maquinaria pesada) y los aspectos culturales (e.g., la percepción diferencial del valor del bosque) (Mastrangelo et al. 2014; Marinaro et al. 2017) determinarían los distintos contextos productivos. El legado de usos anteriores modificaría la composición de las comunidades, los atributos físicos o químicos del suelo y, potencialmente, las propiedades emergentes del ecosistema, como, por ejemplo, la susceptibilidad a los fuegos (Aragón and Morales 2003; Tálamo and Caziani 2003). Finalmente, el tiempo transcurrido desde que se aísla una porción del bosque y el grado de conexión entrelos remanentes determinarían la dinámica poblacional y metapoblacional de las especies nativas (Chacoff et al. 2004; SemperPascual et al. 2018). El esquema de clasificación conceptual presentado en este trabajo (Figura 2) sienta las bases para describir o ponderar particularmente los efectos de la estructura espacial de los remanentes y del tiempo de aislamiento. Las otras dos dimensiones del problema imponen desafíos metodológicos varios, que involucrarían labor de campo tanto sobre el componente humano como natural (e.g., entrevistas a actores locales, estimaciones de carga ganadera) o estimaciones mediante sensores remotos (e.g., discriminación de cultivos y pasturas).
Dada la existencia de fuertes incentivos agronómicos y económicos para deforestar, incluso en las áreas con mayores limitaciones ambientales de la región (Giménez et al. 2015; Murray et al. 2016), la conformación y permanencia de remanentes en paisajes agrícolasestácondicionada fundamentalmente por múltiples factores socio-culturales. Entre ellos destacamos al legislativo, ya que desde principios de la década del 2000 se implementan en la Argentina y el Paraguay distintas normas que regulan u ordenan el uso del territorio (Seghezzo et al. 2011; Collazo et al. 2013). La selección de nuestras AF tuvo en cuenta la Ley $\mathrm{N}^{\circ} 26331$ para la Argentina. En particular, establecimos que estas AF se deberían localizar en áreas de Categoría III (verde), en las que se permite deforestar para cultivar. Sin embargo, existen discrepancias en cuanto al nivel de transformación aceptable entre provincias, tamaños de unidades productivas y aspectos meramente biofísicos (e.g., cercanía a cuerpos de agua). Las diferencias entre AF podrían revelar el impacto de este factor, ya que, por ejemplo, en las AF del Espinal la deforestación habría antecedido a las primeras normas ambientales de comienzos de la década de 1980 (e.g., Ley $\mathrm{N}^{\circ}$ 22428; Senado y Cámara de Diputados de la Nación Argentina 1981) (González-Roglich et al. 2015). No obstante, aun existiendo la legislación, puede ser incumplida, como destacan Ginzburg et al. (2012) para el AF de Charata (nótese la ausencia de cortinas forestales en la Figura 4b).

Otros factores socio-culturales fueron menos explorados. El usuario individual o 
comunitario podría conservar el remanente por una preferencia ideológica, por la capacidad del remanente para proveer bienes o servicios de difícil valoración económica o por no poseer los medios financieros o un título de propiedad regularizado para deforestar. Estos factores se pueden resumir o sintetizar en el tipo de usuario del territorio como, por ejemplo, los sistemas de producción agroindustrial y los campesino/indígenas (Baldi et al. 2015; Marinaro et al. 2015). Los actores campesinos o indígenas perciben y valorizan otros servicios que entrega el bosque, tales como la provisión de leña y carbón, plantas medicinales, forraje o sombra para el ganado (Cáceres et al. 2015; Laclau et al. 2015). Desde lo meramente económico, estos actores rara vez cuentan con una financiación inicial para reemplazar grandes extensiones de vegetación nativa. Fernández et al. (2014) cuantifican que el costo local de la deforestación y de la subsecuente implantación de pasturas superaría los 550 US\$ / ha, y que la inversión inicial se amortizaría a los tres años. Mastrangelo et al. (2014) encontraron que en la provincia de Chaco, los tipos de actores con problemas de tenencia, menor acceso al crédito y menor relación trabajo/consumo identifican y perciben más fuertemente a la deforestación como un problema ambiental.

Al momento de comenzar con la caracterización aquí representada (año 2014), nuestra capacidad de distinguir remanentes se encontraba condicionada por la disponibilidad de imágenes satelitales de una resolución espacial de mayor detalle a la de los productos Landsat 7 u 8 (30 m). Esta disponibilidad se ha ampliado notablemente dado que existen en la actualidad múltiples productos satelitales de acceso parcial o totalmente irrestricto, que permiten caracterizar la cobertura del suelo con un nivel de detalle menor a $10 \mathrm{~m}$. El proyecto CBERS incorporó una cuarta misión que permitiría evaluar la dinámica temporal de los remanentes en nuestra región. Otros productos destacables, como el Sentinel-2A y los SPOT
6/7 (Drusch et al. 2012), ya se emplean para monitorear la cobertura de bosques (Vaglio Laurin et al. 2016). En paralelo, los sensores pasivos o activos montados sobre vehículos aéreos no tripulados (VANT) permiten identificar elementos individuales del dosel y generar modelos digitales de elevación para estimar múltiples atributos estructurales (e.g., Anderson and Gaston 2013; Tuominen et al. 2015) (Figura 5b).

Las decisiones sobre el manejo de remanentes de bosque nativo adquieren gran relevancia en el Chaco Seco y el Espinal dado el fuerte avance agrícola de las últimas dos décadas y la consecuente disminución y fragmentación de la cobertura boscosa (Piquer-Rodríguez et al. 2015). Este manejo debe tener un sustento científico claro y debe contemplar las valorizaciones contrapuestas de distintos actores locales y externos. A partir de este cuerpo de información se debería alimentar a una legislación sólida, consistente entre unidades políticas y respetada por la sociedad. Como ejemplo, las múltiples normas legales que rigen la extensión y la disposición de las cortinas forestales a escala predial buscan históricamente controlar la erosión eólica e hídrica. Sin embargo, al ignorar las pérdidas de la producción y mayores gastos de manejo agronómico, son frecuentemente ignoradas por el productor agropecuario agroindustrial (Ginzburg et al. 2012). En este trabajo brindamos herramientas para la identificación y el monitoreo de estos remanentes, las cuales podrán ser consideradas tanto con fines productivos como de conservación.

Agradecimientos. Este trabajo contó con el apoyo de los subsidios otorgados por la Agencia Nacional de Promoción Científica y Tecnológica PICT 2013-2973 y PICT 20160972 y por el Instituto Interamericano para la Investigación del Cambio Global (IAI) CRN 3095. Agradecemos a R.G. Ginzburg, S. Schauman, N.I. Gasparri y a los dos revisores anónimos por sus valiosos comentarios.

\section{REFERENCIAS}

Adamoli, J., R. Ginzburg, and S. Torrella. 2011. Escenarios productivos y ambientales del Chaco Argentino: 1977-2010. Fundación Producir Conservando, Buenos Aires.

Anderson, K., and K. J. Gaston. 2013. Lightweight unmanned aerial vehicles will revolutionize spatial ecology. Frontiers in Ecology and the Environment 11:138-146.

Aragón, R., and J. M. Morales. 2003. Species composition and invasion in NW Argentinian secondary forests: Effects of land use history, environment and landscape. Journal of Vegetation Science 14:195-204.

Baldi, G., J. P. Guerschman, and J. M. Paruelo. 2006. Characterizing fragmentation in temperate South America grasslands. Agriculture, Ecosystems and Environment 116:197-208.

Baldi, G., J. Houspanossian, F. Murray, A. A. Rosales, C. V. Rueda, and E. G. Jobbágy. 2015. Cultivating the dry forests of South America: Diversity of land users and imprints on ecosystem functioning. Journal of Arid Environments 123:47-59. 
Baldi, G., and E. G. Jobbágy. 2012. Land use in the dry subtropics: Vegetation composition and production across contrasting human contexts. Journal of Arid Environments 76:115-127.

Bicheron, P., M. Huc, C. Henry, and GLOBCOVER partners. 2008. GLOBCOVER: Description Products Manual.

Cabrera, A. L. 1971. Fitogeografía de la República Argentina. Sociedad Argentina de Botánica 14:1-42.

Cáceres, D. M., E. Tapella, F. Quétier, and S. Díaz. 2015. The social value of biodiversity and ecosystem services from the perspectives of different social actors. Ecology and Society 20: 62

Carranza, M. L., L. Hoyos, L. Frate, A. T. R. Acosta, and M. Cabido. 2015. Measuring forest fragmentation using multitemporal forest cover maps: Forest loss and spatial pattern analysis in the Gran Chaco, central Argentina. Landscape and Urban Planning 143:238-247.

Chacoff, N. P., and M. A. Aizen. 2006. Edge effects on flower-visiting insects in grapefruit plantations bordering premontane subtropical forest. Journal of Applied Ecology 43:18-27.

Chacoff, N. P., J. M. Morales, and M. del P. Vaquera. 2004. Efectos de la Fragmentación Sobre la Absorción y Depredación de Semillas en el Chaco Serrano. Biotropica 36:109-117.

Chen, J., J. Chen, A. Liao, X. Cao, L. Chen, X. Chen, C. He, G. Han, S. Peng, M. Lu, W. Zhang, X. Tong, and J. Mills. 2015. Global land cover mapping at $30 \mathrm{~m}$ resolution: A POK-based operational approach. ISPRS Journal of Photogrammetry and Remote Sensing 103:7-27.

Collazo, M. A. G., A. Panizza, and J. M. Paruelo. 2013. Ordenamiento territorial de bosques nativos: Resultados de la zonificación realizada por provincias del norte argentino. Ecología Austral 23:97-107.

Congalton, R. 1991. A Review of assessing the accuracy of classifications of remotely sensed data. Remote Sensing of Environment 37:35-46.

Drusch, M., U. Del Bello, S. Carlier, O. Colin, V. Fernandez, F. Gascon, B. Hoersch, C. Isola, P. Laberinti, P. Martimort, A. Meygret, F. Spoto, O. Sy, F. Marchese, and P. Bargellini. 2012. Sentinel-2: ESA's Optical High-Resolution Mission for GMES Operational Services. Remote Sensing of Environment 120:25-36.

Fernández, D., A. Krysa, and L. Ortega. 2014. El avance de la soja hacia fuera de la región pampeana. Reflexiones sobre el papel de la renta agraria y la política estatal. Octavas Jornadas de Investigadores en Economías Regionales. Editorial Universitaria de la Universidad Nacional de Misiones, Posadas, Argentina.

Forman, R. T. T. 1995. Land Mosaics. The ecology of landscapes and regions. Cambridge University Press, Cambridge, UK.

Garibaldi, L. A., I. Steffan-Dewenter, C. Kremen, J. M. Morales, R. Bommarco, S. A. Cunningham, L. G. Carvalheiro, N. P. Chacoff, J. H. Dudenhöffer, S. S. Greenleaf, A. Holzschuh, R. Isaacs, K. Krewenka, Y. Mandelik, M. M. Mayfield, L. A. Morandin, S. G. Potts, T. H. Ricketts, H. Szentgyörgyi, B. F. Viana, C. Westphal, R. Winfree, and A. M. Klein. 2011. Stability of pollination services decreases with isolation from natural areas despite honey bee visits. Ecology Letters 14:1062-1072.

Gasparri, N. I., and H. R. Grau. 2009. Deforestation and fragmentation of Chaco dry forest in NW Argentina (19722007). Forest Ecology and Management 6:913-921.

Gasparri, N. I., and G. Baldi. 2013. Regional patterns and controls of biomass in semiarid woodlands: lessons from the Northern Argentina Dry Chaco. Regional Environmental Change 13:1131-1144.

Gasparri, N. I., T. Kuemmerle, P. Meyfroidt, Y. le Polain de Waroux, and H. Kreft. 2016. The Emerging Soybean Production Frontier in Southern Africa: Conservation Challenges and the Role of South-South Telecouplings. Conservation Letters 9:21-31.

Giménez, R., J. Houspanossian, J. L. Mercau, and E. G. Jobbágy. 2015. Balancing agricultural and hydrological risk in farming systems of the Chaco plains. Journal of Arid Environments 123:81-92.

Ginzburg, R. G., S. A. Torrella, and J. M. Adamoli. 2012. Las cortinas forestales de bosque nativo, ¿son eficaces para mitigar los efectos de la expansión agrícola? Revista de la Asociación Argentina de Ecología de Paisajes 3:34-42.

González-Roglich, M., J. J. Swenson, D. Villarreal, E. G. Jobbágy, and R. B. Jackson. 2015. Woody Plant-Cover Dynamics in Argentine Savannas from the 1880s to 2000s: The Interplay of Encroachment and Agriculture Conversion at Varying Scales. Ecosystems 18:481-492.

Guyra Paraguay. 2015. Monitoreo de los cambios de uso de la tierra, incendios e inundaciones Gran Chaco Americano. Guyra Paraguay, Asunción, Paraguay.

Hansen, M. C., P. V. Potapov, R. Moore, M. Hancher, S. A. Turubanova, A. Tyukavina, D. Thau, S. V. Stehman, S. J. Goetz, T. R. Loveland, A. Kommareddy, A. Egorov, L. Chini, C. O. Justice, and J. R. G. Townshend. 2013. Highresolution global maps of 21st-century forest cover change. Science 342:850-853.

Hay, G. J., G. Castilla, M. A. Wulder, and J. R. Ruiz. 2005. An automated object-based approach for the multiscale image segmentation of forest scenes. International Journal of Applied Earth Observation and Geoinformation 7:339-359.

Houspanossian, J., R. Giménez, G. Baldi, and M. D. Nosetto. 2016. Is aridity restricting deforestation and land uses in the South American Dry Chaco? Journal of Land Use Science 11:369-383.

Jaeger, J. A. G. 2000. Landscape division, splitting index, and effective mesh size: new measures of landscape fragmentation. Landscape Ecology 15:115-130.

Krauss, J., A. M. Klein, I. Steffan-Dewenter, and T. Tscharntke. 2004. Effects of habitat area, isolation, and landscape diversity on plant species richness of calcareous grasslands. Biodiversity and Conservation 13:1427-1439.

Kunst, C., E. Monti, H. Perez, and J. Godoy. 2006. Assessment of the rangelands of southwestern Santiago del Estero, Argentina, for grazing management and research. Journal of Environmental Management 80:248-265.

Laclau, P., D. Daguer, and G. Caballe. 2015. Sombreado forestal aplicado a tambos. Simulaciones de cortinas forestales. Tree shading in dairy farms. Forest shelterbelts simulations. Pp. 33-36 en Congreso Nacional de Sistemas Silvopastoriles 
/ VIII Congreso Internacional Sistemas Agroforestales. Ediciones INTA, Iguazú, Argentina.

Macchi, L., and H. R. Grau. 2012. Piospheres in the dry Chaco. Contrasting effects of livestock puestos on forest vegetation and bird communities. Journal of Arid Environments 87:176-187.

Marinaro, S., H. R. Grau, N. I. Gasparri, T. Kuemmerle, and M. Baumann. 2017. Differences in production, carbon stocks and biodiversity outcomes of land tenure regimes in the Argentine Dry Chaco. Environmental Research Letters 12:045003.

Marinaro, S., H. R. Grau, L. Macchi, and P. V. Zelaya. 2015. Land tenure and biological communities in dry Chaco forests of northern Argentina. Journal of Arid Environments 123:60-67.

Mastrangelo, M. E., M. C. Gavin, P. Laterra, W. L. Linklater, and T. L. Milfont. 2014. Psycho-Social Factors Influencing Forest Conservation Intentions on the Agricultural Frontier. Conservation Letters 7:103-110.

McGarigal, K., S. A. Cushman, and E. Ene. 2015. Frag. Amherst, USA.

Miles, L., A. C. Newton, R. S. DeFries, C. Ravilious, I. May, S. Blyth, V. Kapos, and J. E. Gordon. 2006. A global overview of the conservation status of tropical dry forests. Journal of Biogeography 33:491-505.

Murray, F., G. Baldi, T. von Bernard, E. F. Viglizzo, and E. G. Jobbágy. 2016. Productive performance of alternative land covers along aridity gradients: Ecological, agronomic and economic perspectives. Agricultural Systems 149:20-29.

Núñez-Regueiro, M. M., L. Branch, R. J. Fletcher Jr, G. A. Marás, E. Derlindati, and A. Tálamo. 2015. Spatial patterns of mammal occurrence in forest strips surrounded by agricultural crops of the Chaco region, Argentina. Biological Conservation 187:19-26.

Piquer-Rodríguez, M., S. Torella, G. Gavier-Pizarro, J. Volante, D. Somma, R. Ginzburg, and T. Kuemmerle. 2015. Effects of past and future land conversions on forest connectivity in the Argentine Chaco. Landscape Ecology 30:817-833.

Redo, D., A. C. Millington, and D. Hindery. 2011. Deforestation dynamics and policy changes in Bolivia's post-neoliberal era. Land Use Policy 28:227-241.

Rempel, R. S., D. Kaukinen, and A. P. Carr. 2012. Patch Analyst and Patch Grid. Ontario Ministry of Natural Resources. Centre for Northern Forest Ecosystem Research, Thunder Bay, Canada.

Riitters, K. H., R. V. O`Neill, C. T. Hunsaker, J. D. Wickham, D. H. Yankee, S. P. Timmins, K. B. Jones, and B. L. Jackson. 1995. A factor analysis of landscape pattern and structure metrics. Landscape Ecology 10:23-39.

Rueda, C. V., G. Baldi, N. I. Gasparri, and E. G. Jobbágy. 2015. Charcoal production in the South American Dry Chaco: Where, how and who? Energy for Sustainable Development 27:46-53.

Saunders, D. A., R. J. Hobss, and C. R. Margules. 1991. Biological consequences of ecosystem fragmentation: a review. Conservation Biology 5:18-32.

Seghezzo, L., J. N. Volante, J. M. Paruelo, D. J. Somma, E. C. Buliubasich, H. E. Rodríguez, S. Gagnon, and M. Hufty. 2011. Native Forests and Agriculture in Salta (Argentina): Conflicting Visions of Development. Journal of Environment \& Development 20:251-277.

Semper-Pascual, A., L. Macchi, F. M. Sabatini, J. Decarre, M. Baumann, P. G. Blendinger, B. Gómez-Valencia, M. E. Mastrangelo, and T. Kuemmerle. 2018. Mapping extinction debt highlights conservation opportunities for birds and mammals in the South American Chaco. Journal of Applied Ecology 55:1218-1229.

Senado y Cámara de Diputados de la Nación Argentina. 1981. Ley N²2.428 de "Fomento a la conservación de los suelos". Boletín Oficial de la República Argentina.

Senado y Cámara de Diputados de la Nación Argentina. 2007. Ley N²6.331 de "Presupuestos Mínimos de Protección Ambiental de los Bosques Nativos". Boletín Oficial de la República Argentina.

Tabarelli, M., A. V. Aguiar, M. C. Ribeiro, J. P. Metzger, and C. A. Peres. 2010. Prospects for biodiversity conservation in the Atlantic Forest: Lessons from aging human-modified landscapes. Biological Conservation 143:2328-2340.

Tálamo, A., and S. M. Caziani. 2003. Variation in woody vegetation among sites with different disturbance histories in the Argentine Chaco. Forest Ecology and Management 184:79-92.

Torrella, S. A., R. G. Ginzburg, J. M. Adamoli, and L. Galetto. 2013. Changes in forest structure and tree recruitment in Argentinean Chaco: Effects of fragment size and landscape forest cover. Forest Ecology and Management 307: 147-154.

Tuominen, S., A. Balazs, H. Saari, I. Pölönen, J. Sarkeala, and R. Viitala. 2015. Unmanned aerial system imagery and photogrammetric canopy height data in area-based estimation of forest variables. Silva Fennica 49:1348.

UMSEF. 2008. Monitoreo de la superficie de bosque nativo de Argentina. Secretaria de Ambiente y Desarrollo Sustentable, Buenos Aires, Argentina.

Vaglio Laurin, G., N. Puletti, W. Hawthorne, V. Liesenberg, P. Corona, D. Papale, Q. Chen, and R. Valentini. 2016. Discrimination of tropical forest types, dominant species, and mapping of functional guilds by hyperspectral and simulated multispectral Sentinel-2 data. Remote Sensing of Environment 176:163-176.

Vallejos, M., J. N. Volante, M. J. Mosciaro, L. M. Vale, M. L. Bustamante, and J. M. Paruelo. 2015. Transformation dynamics of the natural cover in the Dry Chaco ecoregion: A plot level geo-database from 1976 to 2012. Journal of Arid Environments 123:3-11.

van Dam, C. 2003. Cambio tecnológico, concentración de la propiedad y desarrollo sostenible: los efectos de la introducción del paquete soja/siembra directa en el Umbral al Chaco. Debate Agrario 35:133-181.

Vijayaraj, V., C. G. O'Hara, and N. H. Younan. 2004. Quality analysis of pansharpened images. Pp. 85-88 in International Geoscience and Remote Sensing Symposium (IGARSS).

Volante, J. N., D. Alcaraz-Segura, M. J. Mosciaro, E. F. Viglizzo, and J. M. Paruelo. 2012. Ecosystem functional changes associated with land clearing in NW Argentina. Agriculture, Ecosystems and Environment 154:12-22. 